\title{
Medical Management of GDM - following the evidence Mini Commentary on 21-0353.R1 - "Changing Patterns in Medication Prescription for Gestational Diabetes During a Time of Guideline Change in the USA: A Cross-sectional Study"
}

\author{
Aaron Caughey ${ }^{1}$ \\ ${ }^{1}$ Oregon Health and Science University
}

June 24, 2021

\section{Medical Management of GDM - following the evidence \\ Mini Commentary on 21-0353.R1 - "Changing Patterns in Medication Prescription for Gestational Diabetes During a Time of Guideline Change in the USA: A Cross-sectional Study"}

Aaron B. Caughey, MD, PhD

Department of Obstetrics and Gynecology; Oregon Health \& Science University; Portland, OR

Correspondence:

Aaron B. Caughey, MD, PhD

Professor and Chair

Department of Obstetrics and Gynecology

Oregon Health \& Sciences University

P: 503-494-2999

F: 503-494-2391

E: caughey@ohsu.edu

Gestational diabetes mellitus (GDM) is one of the most common pregnancy complications and is associated with numerous pregnancy complications including preeclampsia, preterm birth, stillbirth, cesarean delivery, fetal macrosomia, birth injury, neonatal hypoglycemia, childhood obesity in the offspring and other short- and long-term complications.(Sweeting AN, et al. Diabetes Care. 2016;39:75-81) Many of these complications appear to be associated with hyperglycemia, so tight control of maternal plasma glucose is the primary approach to management during pregnancy. The first line approach is usually a strict carbohydrate-controlled diet and exercise, but when this fails, medical therapy is indicated.

For many years, the first-line medical approach was injectable insulin. However, an oral hypoglycemic agent, glyburide, was increasingly adopted after a trial of glyburide versus insulin demonstrated no statistical difference in the outcomes, though it was underpowered to do so.(Langer O, et al. N Engl J Med 2000;343:1134-8) Another oral agent, metformin, was studied in an adequately powered randomized trial that found no differences in outcomes between pregnancies treated with metformin versus insulin.(Rowan JA, et al. N Engl J Med 2008;358:2003-15) Perhaps because glyburide was already being used, or that metformin crosses the 
placenta, or an odd finding of a higher rate of preterm birth in the metformin group, there was little adoption of metformin after this trial. Other trials of these medications have been conducted and a systematic review in 2015 demonstrated that a number of outcomes were worse in women randomized to glyburide whereas there are no differences in women randomized to metformin.(Balsells M, et al. BMJ 2015;350:h102) These findings led the American College of Obstetricians and Gynecologists (ACOG) to change their recommendations to encourage insulin to be first-line treatment in 2017.(ACOG. Obstet Gynecol. 2017;130:e17-e37) A follow-up recommendation from ACOG broadened to include metformin as well.(ACOG. Obstet Gynecol. 2018 Feb;131(2):e49-e64)

So, how have these data and the recommendations from ACOG changed practice patterns? In a paper published in BJOG this month, the authors examined the pharmacologic approaches to management in GDM.(Venkatesh KK, et al. BJOG, 2021) In a large cohort of over 10,000 individuals with GDM requiring medical therapy, they found that from 2015 to 2018, the use of insulin increased from $26 \%$ to $44 \%$, the use of metformin increased from $17 \%$ to $29 \%$, and the use of glyburide decreased from $58 \%$ to $27 \%$. These data are from either side of the 2017 ACOG recommendation, but end in the same year as the follow-up recommendation. Given how long it can take for recommendations to be adopted by clinicians, one wonders how the practice patterns may have continued to evolve in 2019 and 2020.

Recalling that the 2017 ACOG recommendation was to have insulin be first line for GDM, while the trend is in the right direction, it was still less than $50 \%$ as of 2018 . While it is understandable that clinicians and patients want to prescribe an oral agent, insulin remains an effective medication with great safety data. Likely this is why it is recommended as first-line treatment by many organizations and hopefully, further trends towards its primary use will be seen. 